\title{
A retrospective cohort analysis of the relationship between thyroid hormone level and herpes simplex virus-1 activation
}

\author{
Shao-Chung Victor Hsia*, Jayesh Parmar, Robert Freeman, Matthew Balish \\ School of Pharmacy, University of Maryland Eastern Shore, Princess Anne, USA; *Corresponding Author: vhsia@umes.edu
}

Received 20 February 2013; revised 21 March 2013; accepted 15 April 2013

Copyright (C 2013 Shao-Chung Victor Hsia et al. This is an open access article distributed under the Creative Commons Attribution License, which permits unrestricted use, distribution, and reproduction in any medium, provided the original work is properly cited.

\section{ABSTRACT}

A number of physiological factors have been suggested to participate in the Herpes Simplex Virus Type-1 (HSV-1) reactivation. Of particular interest is the effect of hormonal aberration on gene expression and activation. Thyroid hormone (TH) was shown to play a role in HSV-1 gene expression and replication in cell culture and animal models. We hypothesize that TH participates in the control of HSV latency and reactivation in humans by regulating viral gene expression and replication. Prior to implementing a full-scale population-based inquiry into this hypothesis, a pilot study using a pharmacy claims data base and a case-controlled, retrospective cohort preliminary investigation was conducted to develop further the hypothetical link between TH and HSV-1 reactivation. Using prescriptions for treating thyroid disorders and HSV-1 infections as proxies for biologic functions, we queried a prescription data base to construct two patient cohorts: Cohort 1 was comprised of patients receiving prescription drugs for thyroid disorders over a three-month period, and Cohort 2 was composed of patients not receiving thyroid medications during this period. HSV-1 medications were recorded for each cohort and the difference in the frequency of HSV-1 prescription drug utilization was examined for statistical significance. Using a $2 \times 2$ contingency table, a chi-square of $\mathbf{1 0 . 1 2}$ was calculated that was significant at $p=0.0015$, confirming that a significant difference was found in HSV-1 utilization between these two cohorts, suggesting that patients who receive thyroid drugs have a greater chance of receiving antiviral drugs for HSV-1 infection/reactivation. Since this pilot study has inherent limitations in the data set, this finding is descriptive, not explanatory, and further research involving more detailed patient records in a larger patient population will be implemented to explore the relationship more robustly.

Keywords: HSV-1; Thyroid Hormone; Retrospective Study; Viral Reactivation; Odds Ratio; Pharmacy Claims

\section{INTRODUCTION}

Thyroid hormone ( $\mathrm{TH}$ or $\mathrm{T}_{3}$ ) is known to be important for normal functioning of numerous essential physiological processes and organs. Mechanistically, TH exerts its effects through its nuclear receptor (TR) which binds to the thyroid hormone response element (TRE) located within the genes' regulatory sequence [1]. TR, a transcriptional factor, controls gene expression and the net outcome is determined by TH level [2]. Endocrine dysfunctions have been shown to cause viral infection [3]. Recent studies suggested that $\mathrm{TH}$ and TRs played roles on HSV-1 gene silencing/activation and DNA replication and may have implication during latency/reactivation [4, 5].

HSV-1 is one of the most widespread infections in humans [6]. Seroprevalence rates in the United States suggest that the virus is present in $57.7 \%$ of the population [7]. After the primary acute attack, the virus may establish latency in the sensory neurons of trigeminal ganglia and the reactivation may occur momentarily at the mucosa, lips, eyes, or perioral area of the face causing fever blisters [8]. HSV-1 is responsible for approximately $10 \%$ of viral encephalitis and majority of cases results from reactivation [9]. Other major clinical symptoms include cornea infection (herpes keratitis) [10] genital HSV in- 
fection [11] and HSV-2, or genital herpes. The molecular/cellular modes of action leading to HSV reactivation are unclear [12].

Case reports indicated that HSV may reactivate following episodes of stress, injury to tissues innervated by neurons harboring the latent virus or other local or systemic stimuli thus it is of interest that many factors triggered HSV-1 reactivation reduced TH levels as well [3]. Based on these in vivo and in vitro studies, it is hypothesized that $\mathrm{TH}$ status is associated in determining HSV-1 latency and reactivation. In effect, hypothyroidism relieves TR-mediated inhibition and induced viral gene expression and replication. Nonetheless, there is no controlled clinical evidence in human subjects supporting this hypothesis. The lack of a priori empiric evidence in humans between thyroid hormone abnormalities and the subsequent activation of HSV represents a challenge to justifying an extension of the hypothesis beyond those from the in vitro and in vivo results.

\section{MATERIALS AND METHODS}

In order to corroborate the working hypothesis from pre-clinical evidence to that which might be found in human subjects, a case-control, retrospective pilot study using a prescription drug claims data base was conducted. In essence, this type of quasi-experimental research design was chosen as an initial step in the further development of the hypothesis that abnormal thyroid function is related to activation of the HSV. In particular, this design permits an initial exploration of the odds that utilization of thyroid medications is associated with HSV reactivation as measured by antiviral drug utilization.

Three-month prescription utilization of thyroid medications and antiviral drugs were obtained from a regional chain pharmacy's 2012 claims data base of 8474 prescriptions. Of the estimated 2690 patients receiving prescriptions, the following drug specific utilization data were found: 1) total number of prescriptions for all medications dispensed (8474); 2) number of patients without prescriptions for antivirals acyclovir, famciclovir, penciclovir, valacyclovir, and gancicovir\}and thyroid medications \{Levothyroxine and thyroid desiccated (2109); 3) number of patients with prescriptions for thyroid medications (469); 4) number of patients with prescriptions for thyroid medications and no (0) antiviral medications (461); 5) number of patients with prescriptions for antiviral medications (120); 6) number of patients receiving antiviral medications with no prescriptions (0) for thyroid medications (112); and 7) number of patients receiving thyroid medications AND antiviral medications [8].

From these data, two cohorts were constructed (Figure 1): Cohort 1: Patients receiving at least one prescription for a thyroid medication during the three-month period; and Cohort 2: Patients who did not receive a prescription for a thyroid medication during the three-month
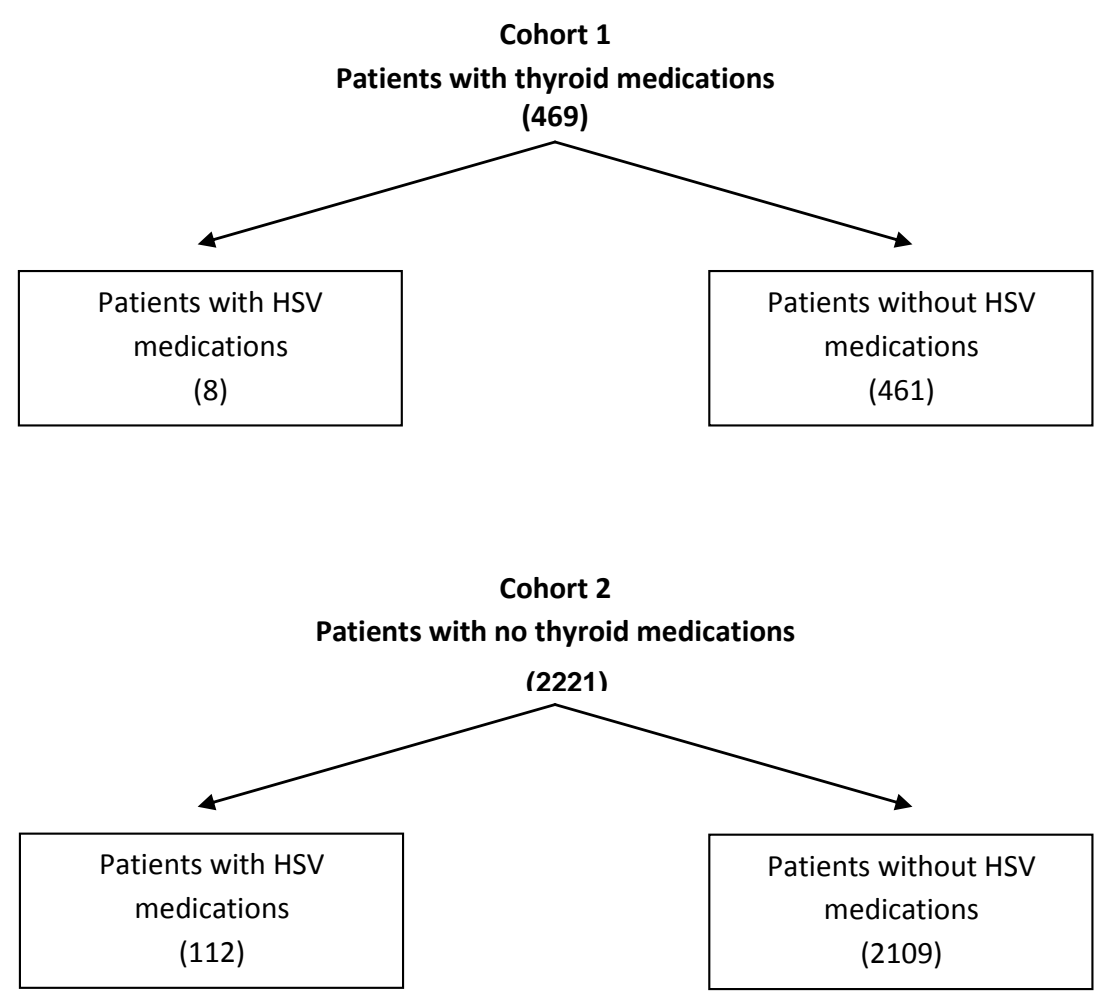

Figure 1. Number of patients in cohort 1 and cohort 2. 
period. Prescriptions for antiviral medications were recorded for each cohort over the same period. It was assumed that Cohort 1 was comprised of patients with a thyroid disorder, whereas Cohort 2 was comprised of patient with normal thyroid function. If a thyroid disorder reactivates $\mathrm{HSV}-1$, then a significant difference in the utilization of antiviral drugs should be evident between the two cohorts.

For the calculated chi-square, acceptance of the null hypothesis would suggest that there is no difference between antiviral drug utilization between the two cohorts, and consequently, that thyroid disorders are not associated with activation of HSV. Conversely, a significant $p$-value would suggest a correlation between thyroid disorders and HSV activation, as defined by the proxy measure of drug utilization.

\section{RESULTS}

A $2 \times 2$ contingency table (Table 1 ) was set up for statistical analyses to investigate the relationship between TH level and HSV-1 reactivation. The results showed that we obtained a chi-square of 10.12 and $p$ value of 0.001467 .

As noted, the calculated chi-square of 10.12 is statistically significant at $p$ value of 0.0015 . This suggests that patients who receive thyroid drugs have an increased likelihood of receiving antiviral drugs for HSV-1 reactivation. A significant Odds Ratio was observed indicating a greater than three-fold increase in the likelihood of receiving an antiviral drug in the abnormal thyroid cohort.

Table 1. The total number of prescription over three consecutive months in 2012 is 8474 . The average number of retail prescriptions per capita is estimated to be $12.6^{1}$. The average number of retail prescriptions per quarter is calculated to be 3.15. Therefore, total number of patients with medications per quarter $=8474 / 3.15=2690$.

\begin{tabular}{|c|c|c|c|c|}
\hline \multirow{4}{*}{$\begin{array}{l}\text { Number of } \\
\text { patients } \\
\text { with HSV } \\
\text { medications }\end{array}$} & \multicolumn{4}{|c|}{$\begin{array}{l}\text { Number of patients with } \\
\text { thyroid medications }\end{array}$} \\
\hline & & Yes & No & Total \\
\hline & Yes & 8 & 112 & 120 \\
\hline & No & 461 & 2109 & 2570 \\
\hline & Total & 469 & 2221 & 2690 \\
\hline Analyses Result & $p$ value & 0.0015 & chi-square & 10.12 \\
\hline
\end{tabular}

${ }^{1}$ Kaiser Family Foundation calculations using data from IMS Health, http://www.imshealth.com (Press Room, US Top-Line Industry Data 2008), and Census Bureau, http://www.census.gov. The per capita number may differ from the number reported at KFF's website www.statehealthfacts.org because of differing data sources which use different retail pharmacy definitions (e.g., IMS Health includes mail order, Verispan does not).

\section{DISCUSSION}

\subsection{Clinical Relevance: Abnormality of TH Level and HSV-1 Reactivation}

There are reasons to hypothesize that the status of $\mathrm{TH}$ can influence HSV-1 reactivation and latency. First, it has been reported that $\mathrm{TH}$ interacted with its nuclear receptor TRs exert regulatory function on key HSV-1 genes $[4,5]$. Second, TH and TR are present in ganglia neurons $[13,14]$ and can influence the survival, differentiation, maturation of neurons [15] thus affecting the HSV-1 latency status. Third, $\mathrm{TH}$ is vital in controlling axonal transport which is critical for HSV-1 movement during reactivation since $\mathrm{TH}$ and nerve growth factor (NGF) enhanced neurite outgrowth and regulate the expression of dynein, a key protein that is involved in axonal transport, in ganglia neurons [16]. Fourth, it is known that $\mathrm{TH}$ participated in the modulation of innate and adaptive immune responses such as chemotaxis, phagocytosis, production of reactive oxygen species, and synthesis and release of cytokines $[17,18]$. TH can promote human B cell differentiation [19], NK cell activity $[20,21]$, and the sensitivity of lymphocytes to interferons (IFNs) and interferon- mediated cytotoxic activity of NK cells [22-24]. In summary, the modulation of immune system by $\mathrm{TH}$ may significantly affect the viral reactivation.

A number of episodes that decrease TH levels appear to initiate HSV-1 reactivation as well [3]. For example, alteration of corticosteroid has been linked to HSV-1 reactivation $[25,26]$ and a case study showed that a patient with myxedema coma under corticosteroid treatment developed herpes simplex encephalitis with extremely low thyroxine level less than $5.2 \mathrm{nmol} / \mathrm{L}$ (normal range 12 - $30 \mathrm{nmol} / \mathrm{L}$ ) [27]. However, there is no direct, controlled clinical or epidemiological study regarding the effect of TH on HSV-1 reactivation.

Before employing a large-scale population-based examination into this hypothesis, we performed this pilot study using a pharmacy claims data base and a casecontrolled, retrospective cohort survey to examine this hypothetical connection between $\mathrm{TH}$ and HSV-1 reactivation. Prescriptions for treatment of thyroid disorders and HSV-1 infections were cross-examined as proxies for their correspondent biologic functions.

\subsection{Limitations}

The relationship described by this analysis relies on a proxy variable, drug utilization, and in the absence of laboratory values, the findings should be interpreted with the following limitations of both the data and quasi-research design:

1) Case-control cohorts require robust statistical analy- 
ses of relevant variables to provide assurances of equivalency at base line. The data set did not contain standard categorical variables such as patient age, gender and ethnicity, which are commonly examined at the time cohorts are created. There is an absence of information about base line equivalency that cannot be resolved in the pilot study.

2) There is no assurance of temporal ordering; i.e., the thyroid prescription was dispensed prior to the antiviral drug's dispensing. This is a critical assumption to the hypothesis that a thyroid disorder activates HSV-1.

3) Diagnostic information in pharmacy claims data bases are not available. There is no assurance that the medications were related to thyroid disorders and HSV, respectively. In addition, anti-viral drugs are not specific to HSV-1 since they were prescribed to all alphaherpesviruses including HSV-1, HSV-2, and varicella zoster virus (VZV).

There is a conservative bias in this data set; however, that would tend to underestimate the correlation between thyroid function and HSV activation. First, HSV-1 is a medical condition for which patient self-medication with Over-The-Counter (OTC) medications is a treatment option. For this data set, it could be assumed that only those HSV-1 cases that are sufficiently severe for the patient to take a prescription antiviral are represented. This observation would suggest that the incidence rate of HSV-1activation is under-reported, which, in turn, probably understates the strength of the correlation between thyroid function and HSV activation. It is also likely that the estimated number of patients receiving prescription drugs is over estimated. The data set does not distinguish between new prescriptions and refilled prescriptions at the patient's level. Thusly, any patient who received a new prescription at the beginning of the three-month cycle is likely to have had two refills of the initial prescription for each of the two consecutive months. It is not possible to make an adjustment in the formula to estimate total number of patients, the net effect of which is an over estimation.

\subsection{Conclusion}

Our investigation suggested that thyroid hormone status may have implication to affect HSV-1 latency and reactivation. If the link between TH and HSV-1 reactivation holds up upon further basic science and populationbased research, TH levels can become a standard biomarker for both new and recurrent HSV-1 cases. As such, patients can be advised of their relative risk of HSV-1 occurrence and medically treated as necessary. The results of the pilot study add corroboration to the hypothetical link between abnormal thyroid function and reactivation of the HSV-1 in humans. Accordingly, further population-based research is underway to quantify more precisely the nature of the relationship.

\section{ACKNOWLEDGEMENTS}

We acknowledge the support from the Department of Pharmacy Practice at the University of Maryland Eastern Shore, School of Pharmacy. SVH is further supported by NINDS/NIH R01NS081109.

\section{REFERENCES}

[1] Lazar, M.A. (1993) Thyroid hormone receptors: Multiple forms, multiple possibilities. Endocrine Reviews, 14, 184193.

[2] Hsia, S.C. and Shi, Y.B. (2002) Chromatin disruption and histone acetylation in regulation of the human immunodeficiency virus type 1 long terminal repeat by thyroid hormone receptor. Molecular and Cellular Biology, 22, 4043-4052. doi:10.1128/MCB.22.12.4043-4052.2002

[3] Hsia, S.C., Bedadala, G.R. and Balish, M.D. (2011) Effects of thyroid hormone on HSV-1 gene regulation: Implications in the control of viral latency and reactivation. Cell Bioscience, 1, 24. doi:10.1186/2045-3701-1-24

[4] Bedadala, G.R., Pinnoji, R.C., Palem, J.R. and Hsia, S.C. (2010) Thyroid hormone controls the gene expression of HSV-1 LAT and ICP0 in neuronal cells. Cell Research, 20, 587-598. doi:10.1038/cr.2010.50

[5] Hsia, S.C., Pinnoji, R.C., Bedadala, G.R., Hill, J.M. and Palem, J.R. (2010) Regulation of herpes simplex virus type 1 thymidine kinase gene expression by thyroid hormone receptor in cultured neuronal cells. Journal of NeuroVirology, 16, 13-24. doi:10.3109/13550280903552412

[6] Taylor, T.J., Brockman, M.A., McNamee, E.E. and Knipe, D.M. (2002) Herpes simplex virus. Front Bioscience, 7, d752-764. doi:10.2741/taylor

[7] Xu, F., Sternberg, M.R., Kottiri, B.J., McQuillan, G.M., Lee, F.K., Nahmias, A.J., Berman, S.M. and Markowitz, L.E. (2006) Trends in herpes simplex virus type 1 and type 2 seroprevalence in the United States. JAMA, 296, 964-973. doi:10.1001/jama.296.8.964

[8] Hill, J.M., Wen, R. and Halford, W.P. (1998) Pathogenesis and molecular biology of HSV latency and ocular reactivation in the rabbit. Methods in Molecular Medicine, 10, 291-315.

[9] Martinez, P.A., Diaz, R., Gonzalez, D., Oropesa, L., Gonzalez, R., Perez, L., Viera, J. and Kouri, V. (2007) The effect of highly active antiretroviral therapy on outcome of central nervous system herpesviruses infection in Cuban human immunodeficiency virus-infected individuals. Journal of NeuroVirology, 13, 446-451. doi:10.1080/13550280701510088

[10] Shah, A., Farooq, A.V., Tiwari, V., Kim, M.J. and Shukla, D. (2010) HSV-1 infection of human corneal epithelial cells: Receptor-mediated entry and trends of re-infection. Molecular Vision, 16, 2476-2486.

[11] Jonsson, M.K. and Wahren, B. (2004) Sexually transmitted herpes simplex viruses. Scandinavian Journal of Infectious Diseases, 36, 93-101. 
doi:10.1080/00365540310018905

[12] Goel, N., Mao, H., Rong, Q., Docherty, J.J., Zimmerman, D. and Rosenthal, K.S. (2002) The ability of an HSV strain to initiate zosteriform spread correlates with its neuroinvasive disease potential. Archives of Virology, 147, 763-773. doi:10.1007/s007050200024

[13] Walter, I.B. and Droz, B. (1995) Nuclear and cytoplasmic triiodothyronine-binding sites in primary sensory neurons and Schwann cells: Radioautographic study during development. Journal of Neuroendocrinology, 7, 127-136. doi:10.1111/j.1365-2826.1995.tb00675.x

[14] Glauser, L. and Barakat Walter, I. (1997) Differential distribution of thyroid hormone receptor isoform in rat dorsal root ganglia and sciatic nerve in vivo and in vitro. Journal of Neuroendocrinology, 9, 217-227. doi:10.1046/j.1365-2826.1997.d01-1088.x

[15] Walter, I.B. (1996) Triiodothyronine exerts a trophic action on rat sensory neuron survival and neurite outgrowth through different pathways. European Journal of Neuroscience, 8, 455-466. doi:10.1111/j.1460-9568.1996.tb01229.x

[16] Barakat-Walter, I. and Riederer, B.M. (1996) Triiodothyronine and nerve growth factor are required to induce cytoplasmic dynein expression in rat dorsal root ganglion cultures. Developmental Brain Research, 96, 109-119.

[17] De Vito, P., Balducci, V., Leone, S., Percario, Z., Mangino, G., Davis, P.J., Davis, F.B., Affabris, E., Luly, P., Pedersen, J.Z. and Incerpi, S. (2012) Nongenomic effects of thyroid hormones on the immune system cells: New targets, old players. Steroids, 77, 988-995. doi:10.1016/j.steroids.2012.02.018

[18] De Vito, P., Incerpi, S., Pedersen, J.Z., Luly, P., Davis, F.B. and Davis, P.J. (2011) Thyroid hormones as modulators of immune activities at the cellular level. Thyroid, 21, 879-890. doi:10.1089/thy.2010.0429

[19] Paavonen, T. (1982) Enhancement of human B lympho- cyte differentiation in vitro by thyroid hormone. Scandinavian Journal of Immunology, 15, 211-215. doi:10.1111/j.1365-3083.1982.tb00640.x

[20] Provinciali, M., Muzzioli, M., Di Stefano, G. and Fabris, N. (1991) Recovery of spleen cell natural killer activity by thyroid hormone treatment in old mice. Natural Immunity and Cell Growth Regulation, 10, 226-236.

[21] Provinciali, M., Muzzioli, M. and Fabris, N. (1987) Thyroxine-dependent modulation of natural killer activity. Journal of Experimental Pathology, 3, 617-622.

[22] Provinciali, M. and Fabris, N. (1990) Modulation of lymphoid cell sensitivity to interferon by thyroid hormones. Journal of Endocrinological Investigation, 13, 187-191.

[23] Lin, H.Y., Yen, P.M., Davis, F.B. and Davis, P.J. (1997) Protein synthesis-dependent potentiation by thyroxine of antiviral activity of interferon-gamma. American Journal of Physiology, 273, C1225-1232.

[24] Lin, H.Y., Thacore, H.R., Davis, P.J. and Davis, F.B. (1994) Thyroid hormone potentiates the antiviral action of interferon-gamma in cultured human cells. The Journal of Clinical Endocrinology \& Metabolism, 79, 62-65. doi:10.1210/jc.79.1.62

[25] Marquart, M., Bhattacharjee, P., Zheng, X., Kaufman, H., Thompson, H., Varnell, E. and Hill, J. (2003) Ocular reactivation phenotype of HSV-1 strain F(MP)E, a corticosteroid-sensitive strain. Current Eye Research, 26, 205209. doi:10.1076/ceyr.26.3.205.14890

[26] Higaki, S., Gebhardt, B.M., Lukiw, W.J., Thompson, H.W. and Hill, J.M. (2002) Effect of immunosuppression on gene expression in the HSV-1 latently infected mouse trigeminal ganglion. Investigative Ophthalmology \& Visual Science, 43, 1862-1869.

[27] Doherty, M.J., Baxter, A.B. and Longstreth Jr., W.T. (2001) Herpes simplex virus encephalitis complicating myxedema coma treated with corticosteroids. Neurology, 56, 1114-1115. doi:10.1212/WNL.56.8.1114 